\title{
Estimating personal energy expenditure with location data
}

\author{
Simon Hay \\ Computer Laboratory \\ University of Cambridge \\ Cambridge, United Kingdom \\ Email:sjeh3@cam.ac.uk
}

\author{
Stamatina Th. Rassia \\ The Martin Centre \\ for Architectural and Urban Studies \\ University of Cambridge \\ Cambridge, United Kingdom \\ Email:sr414@cam.ac.uk
}

\author{
Alastair R. Beresford \\ Computer Laboratory \\ University of Cambridge \\ Cambridge, United Kingdom \\ Email:arb33@cam.ac.uk
}

\begin{abstract}
Human inactivity has been associated with the incidence of a number of health conditions and chronic diseases, while our increasing energy consumption is a welldocumented problem. A Personal Energy Meter might help identify areas for improvement in our lifestyles that would benefit both our personal health and the global environment. As one strand of this, we explore the possibility of estimating our own energy expenditure from movement traces provided by location systems. This technique offers a number of advantages over accepted accelerometer-based devices. We validate a model which we then apply to analyse the physical activity and working patterns of a total of 60 individuals spread across two separate offices.
\end{abstract}

Keywords-energy measurement; biomedical measurements

\section{INTRODUCTION}

Modern society is making increasingly unsustainable demands on our environment. Meanwhile, our sedentary lifestyles are creating health problems. There is a clear reciprocity between personal and planetary wellbeing-those actions which improve our own health often also have a positive environmental impact-and comprehensive and accurate sensor data will be very useful in ameliorating problems with both of these. Pervasive computing technologies could help provide users with valuable information on their health, physical activity and carbon footprint.

As part of the wider Computing for the Future of the Planet research theme [1], which seeks in part to explore how computers and sensors can allow us to optimise our daily activities and lives, we envisage a Personal Energy Meter (PEM) which can record and apportion an individual's energy usage in order to provide baseline information and incentives for reducing our environmental impact [2]. This encompasses 'energy' in all its forms, from transportation to human effort. It is often difficult to identify potential areas for improvement in our lifestyles, and to assess the effect of any changes we make; we hope that a PEM will help address these problems.

A PEM is a pervasive computing device, since it needs to collect, store, process and share sensor data in order to function. We believe that the same sensor data used to measure the sustainability of our lifestyle can often also be used to assess the personal healthiness of our daily routine. For example, cycling to work has both planetary and personal health benefits.

Consequently, an important use of the PEM is to measure and interpret the energy we expend ourselves through physical activity. A sedentary lifestyle has been linked to obesity [3] and a range of long-term ailments including respiratory deficiencies, coronary heart disease, type II diabetes, colon cancer and osteoporosis [4], [5]. It has been shown that increased physical activity is associated with a lower risk of mortality, and "promoting the potential benefits of physical activity undertaken at home and during exercise may be an important public health message for ageing populations" [6].

Pervasive healthcare seeks to apply the technologies and principles of ubiquitous computing to improve an individual's health. Location data is arguably the most frequently collected piece of contextual information in pervasive computing, and can be used in the PEM to provide a variety of healthcare applications. In this paper we explore what fine-grained location data can tell us about the energy expenditure, and consequently, the health of an individual. This technique offers a number of improvements over the accepted method, which involves dedicated accelerometerbased devices worn on the hip:

- an individual does not suffer the financial cost and physical inconvenience of wearing a specialised activity monitoring device;

- it is possible to determine where and with whom the most energy is expended, helping to identify locations and social groups that encourage physical activity; and

- working hours and patterns are represented in the data, providing an insight into important factors in physical and mental health.

We make three main contributions:

- drawing on prior work, we refine a model to convert fine-grained location data into an estimate of human energy expenditure;

- we validate this model by comparing our locationbased energy estimates to measurements taken from 15 individuals wearing an activity monitor frequently used 
in the medical community for one week; and

- we apply our energy model to provide estimates of the energy expenditure, over one working week, from a total of 60 individuals spread across two separate offices.

We visualise the activity of all the office users to highlight trends and outliers, and discuss the potential of this technique for future pervasive healthcare applications as well as the wider implications for personal energy metering.

\section{ENERGY CONSUMPTION MODEL}

In previous work, we developed a model for estimating energy expenditure based only on location information and derived velocity [7]. For clarity, we summarise the main components.

Ralston showed that, during level walking, the energy expenditure is a linear function of the square of the speed [8]; converting his empirically derived formula to SI units, we obtain:

$$
P_{w} \approx\left(2.02+1.33 v^{2}\right) \cdot m
$$

where $P_{w}$ is power in $\mathrm{W}, v$ is velocity in $\mathrm{m} / \mathrm{s}$ and $m$ is mass in $\mathrm{kg}$.

Our first study location did not include stairs, but these represent a very significant fraction of an individual's indoor energy expenditure. When a person climbs stairs, they must gain gravitational potential energy. This can be expressed as:

$$
E_{g}=m \cdot g \cdot \Delta h
$$

where $E_{g}$ is energy expenditure in $\mathbf{J}, m$ is mass in $\mathrm{kg}, g$ is the acceleration due to gravity $\left(9.81 \mathrm{~m} / \mathrm{s}^{2}\right)$ and $\Delta h$ is the change in height in $\mathrm{m}$. The same formula also captures standing up. Note that we do not take into account energy required to accelerate vertically at this stage and only consider positive changes in height; empirical analysis of the data correlated with Actigraph readings show that these have minimal impact on energy expenditure and they are omitted for the sake of simplicity.

When a person accelerates, they must gain kinetic energy. This can be expressed as:

$$
E_{k}=\frac{1}{2} \cdot m \cdot(\Delta v)^{2}
$$

where $E_{k}$ is energy expenditure in $\mathbf{J}, m$ is mass in $\mathrm{kg}$ and $\Delta v$ is the change in velocity in $\mathrm{m} / \mathrm{s}$.

\section{VALIDATION}

To validate this model, eighteen participants in a study wore both a location device and a GTIM Actigraph for a week. The use of these monitors received ethical approval and the participants' consent. Figure 1 shows a floor plan of the section of the William Gates Building where the participants work, which is fitted with the Bat system [9]. This provides location information in three dimensions accurate

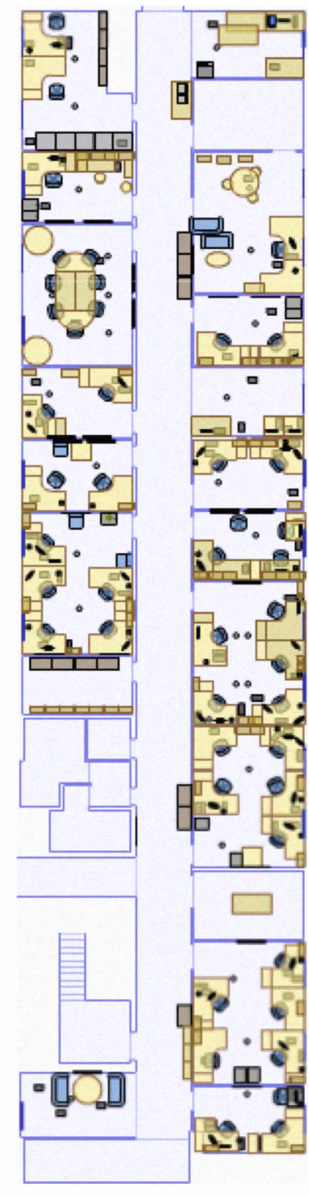

Figure 1. Portion of second floor, William Gates Building

to within $3 \mathrm{~cm} 95 \%$ of the time [10], while the Actigraph is a triaxial accelerometer-based device which previous medical studies have shown provides a reliable record of energy expenditure [11], [12].

The Actigraph records counts which are a dimensionless unit, but Freedson et al. suggest a popular empiricallyderived equation to derive energy expenditure estimates [13]. Converting to SI units for comparison with our model, we obtain:

$$
P=\frac{(0.00094(60 c)+0.1346 m-7.737418) \cdot 4184}{60}
$$
kg.

where $P$ is power in $\mathrm{W}, c$ is counts/s and $m$ is mass in

The Actigraph manual recommends using the Freedson equation only when c exceeds a certain threshold since it not capture the Basal Metabolic Rate (BMR). The Ralston equation used to derive energy expenditure from movement gives a stationary power consumption of approximately $2.02 \mathrm{~m}$ (where $\mathrm{m}$ is mass in $\mathrm{kg}$ ). Accordingly, where $c$ is small we interpolate linearly between an estimate of stationary power consumption taken from the Ralston equation and the value 


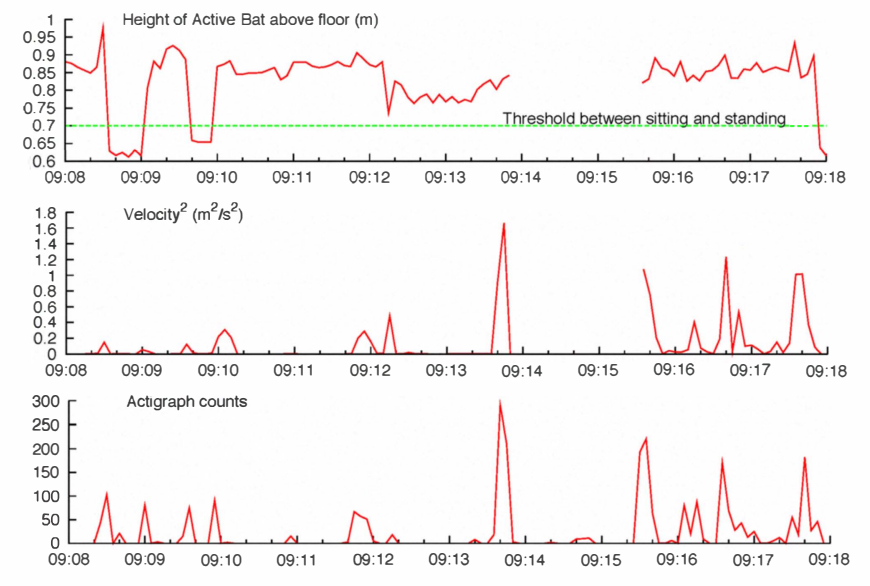

Figure 2. Bat system data correlates closely with Actigraph counts

given by the Freedson equation at the threshold; see [7] for full details.

Figure 2 shows the close correlation between the data that prompted the development of our energy model, while the 'S-curves' in Figure 3 summarise the differences between the energy expenditures recorded by the Actigraph and the estimates from our movement model for each person along with a mean error curve. The $y$ axis shows the percentage of minute-long time periods for which our estimate of energy expenditure differed from that of the Actigraph by less than the value on the $x$ axis. The curves are clustered reasonably tightly, and reveal that, on average, over the course of a week, $90 \%$ of the energy expenditure estimates from the movement model differ from Actigraph data by less than 340 J per minute, while the mean error was $198 \mathrm{~J}$ per minute. For comparison, a person using 2000 calories a day would expend on average around $5811 \mathrm{~J}$ per minute. Of course, it is not known which set of figures are actually closer to the truth; there is some dissatisfaction with the accuracy of the current methods of accelerometer-based monitoring [14], and we believe that our model's output is sufficiently accurate that it can usefully be applied to other studies.

\section{AT\&T LABORATORIES STUDY}

Having validated our model, we can use it to obtain activity estimates from datasets where users did not wear specialised medical devices. The Bat system was originally developed at AT\&T Laboratories Cambridge and all staff (and many visitors) wore the devices continuously for several months, using them to drive a plethora of location-based services, including access to secure areas and have their computer desktops 'follow' them automatically from machine to machine. The movement traces were all recorded, and this dataset therefore provides an excellent opportunity to put our activity estimation model into practice. Figure 4 shows the plan of one floor of Keynes House, where the

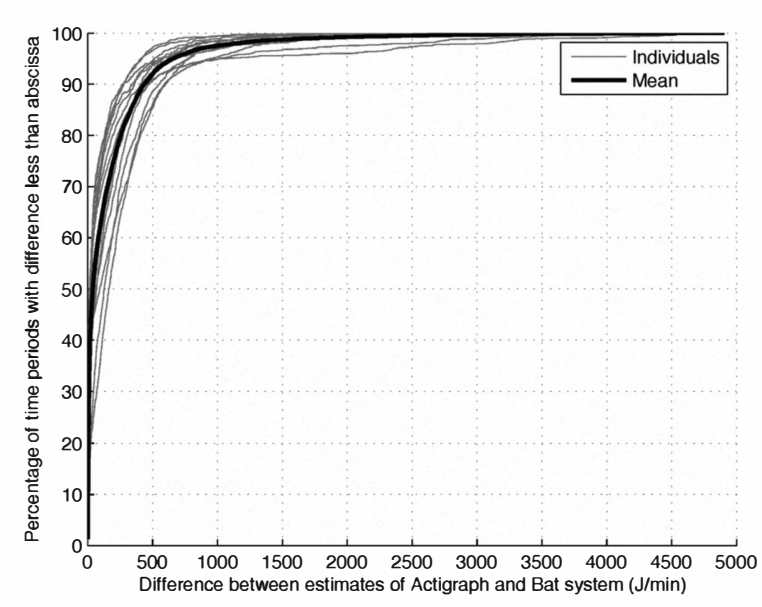

Figure 3. Distributions of differences between energy expenditure estimates from the Actigraph and Bat system data for the William Gates Building over minute-long samples

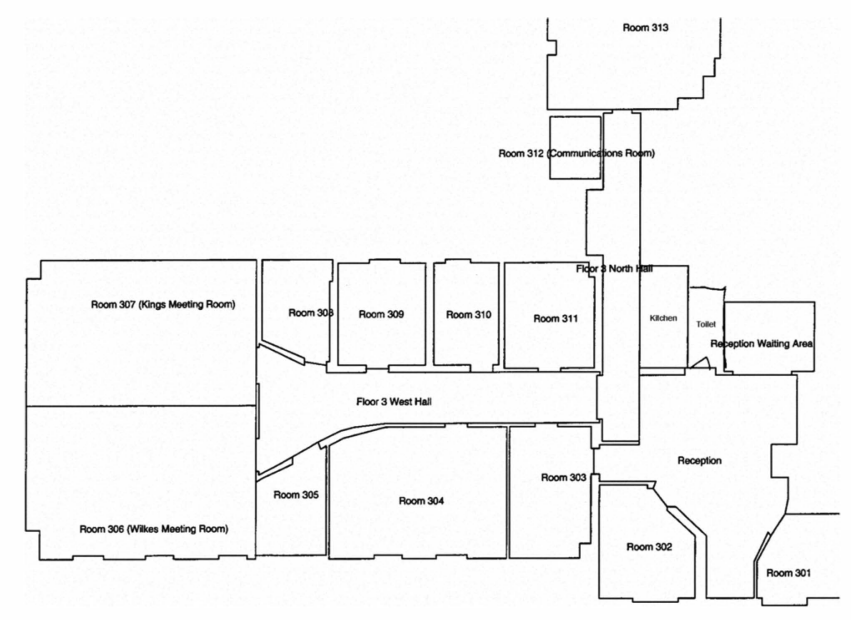

Figure 4. Third floor, Keynes House

laboratories were located; one important difference with this site is that it is split over three floors, and ascending stairs has a significant impact on energy expenditure.

\section{ENERGY EXPENDITURE}

To better understand and compare the data obtained, we devised a 'heat map' visualisation that allows us to represent each user's activity graphically. Figure 5 shows the energy consumption of the users in the Computer Laboratory during our validation study, while Figure 6 shows the top 40 users at AT\&T Laboratories over the course of a typical working week. Each participant has one row, while the $x$ axes represents time, covering Monday morning to Friday evening. Darker colours indicate more intense activity; note that the figure is plotted using a logarithmic scale. Significant variations in the intensity of activity become readily apparent. 
This representation has a number of advantages compared to standard graphs:

- both trends and outliers are highlighted;

- it can be 'zoomed' arbitrarily, providing an at-a-glance overview while also allowing individual analysis;

- it facilitates comparisons amongst a population since its compact form allows a relatively large number of users to be plotted on common axes

- periods with no data, when users are out of the office, also stand out, enabling an analysis of working hours in addition to exploring patterns of physical activity.

\section{DISCUSSION}

The depictions of activity in Figures 5 and 6 reveal a number of traits of interest about the lifestyles of the building occupants. Apart from the total physical activity, which has obvious ramifications enumerated in countless articles of medical research, they show each individual's working hours and patterns. Users working late into the night, or skipping lunch, stand out clearly from their neighbours. How long people work, how regularly they take breaks and their degree of interaction with colleagues all have a demonstrated impact on physical and mental wellbeing.

The rows in Figure 6 are sorted by total activity, so adjacent users have comparable levels of overall energy expenditure-but often show quite different patterns, with some consisting of short bursts of intense activity interspersed with long periods of inactivity while others maintain a more-or-less constant level. Several rows show isolated vertical deep red lines breaking up the normal level of activity; cross referencing these to the original location data show they often represent climbing a flight stairs.

\section{RELATED WORK}

The state of the art in physical activity monitoring is generally based on accelerometers or heart rate sensing [15]. Corder et al. provide a review that examines recent literature on the validation of movement sensors to assess habitual activity [16]. In general, there has been little research on estimating physical activity through movement traces, though the ubiquitous computing field has invested significant effort in mechanisms for obtaining these traces [17].

The Personal Environmental Impact Report is an interesting parallel to the Personal Energy Meter concept that uses location data sampled from mobile phones to calculate personalised estimates of environmental impact and exposure [18]. It is unusual in considering the effect of travel on the individual's health, assessing the user's exposure to smog and even fast food restaurants en route, but does not make an attempt to calculate personal energy expenditure.

\section{FUTURE WORK AND CONCLUSIONS}

Although the location data in these studies was obtained using a highly accurate indoor tracking system, clearly the majority of buildings are not equipped with such technology. It would be interesting to investigate the effect on accuracy of using less fine-grained data, such as might be provided by the sorts of coarse tracking systems that can be deployed by repurposing existing infrastructure [19] or by GPS traces outdoors. This would allow us to extend the model to incorporate additional means of transport, such as cycling.

In this paper, we have outlined a model for estimating physical activity using location data, validated our model against equivalent data from current accepted medical devices and used it to present results from two separate studies of office populations. Pervasive computing systems can provide users with valuable information about their energy expenditure and consumption on both a personal and planetary scale: walking to work instead of driving reduces your overall energy consumption but increases your personal energy expenditure, both of which have a beneficial effect. Our steps here towards one strand of energy information can form a component of a Personal Energy Meter [20], which we hope might motivate lifestyle changes, identify opportunities for improvements and demonstrate the impact that alternatives can have not only on public health but also on the sustainability of our environment.

\section{ACKNOWLEDGEMENTS}

We would like to thank Nick Baker for his initial suggestions and assistance and Andy Hopper for his insight and support.

\section{REFERENCES}

[1] A. Hopper and A. Rice, "Computing for the future of the planet," Philosophical Transactions of the Royal Society A: Mathematical, Physical and Engineering Sciences, vol. 366, no. 1881 , pp. 3685-3697, Oct 2008. [Online]. Available: http://dx.doi.org/10.1098/rsta.2008.0124

[2] S. Hay, "A global personal energy meter," Adjunct Proceedings of the 7th International Conference on Pervasive Computing, 2009. [Online]. Available: http://www.cl.cam.ac. $\mathrm{uk} / \sim \mathrm{sjeh} 3 / \mathrm{pem} /$

[3] U. Ekelund, S. Brage, H. Besson, S. Sharp, and N. J. Wareham, "Time spent being sedentary and weight gain in healthy adults: reverse or bidirectional causality?" Am J Clin Nutr, vol. 88, no. 3, pp. 612-7, Sep 2008. [Online]. Available: http://www.ajen.org/cgi/content/full/88/3/612

[4] S. G. Wannamethee, A. G. Shaper, and M. Walker, "Physical activity and mortality in older men with diagnosed coronary heart disease," Circulation, vol. 102, no. 12, pp. 1358-63, Sep 2000. [Online]. Available: http://circ.ahajournals.org/cgi/content/full/102/12/1358

[5] M. J. Stampfer, F. B. Hu, J. E. Manson, E. B. Rimm, and W. C. Willett, "Primary prevention of coronary heart disease in women through diet and lifestyle," $N$ Engl J Med, vol. 343, no. 1, pp. 16-22, Jul 2000. [Online]. Available: http://content.nejm.org/cgi/content/abstract/343/1/16 


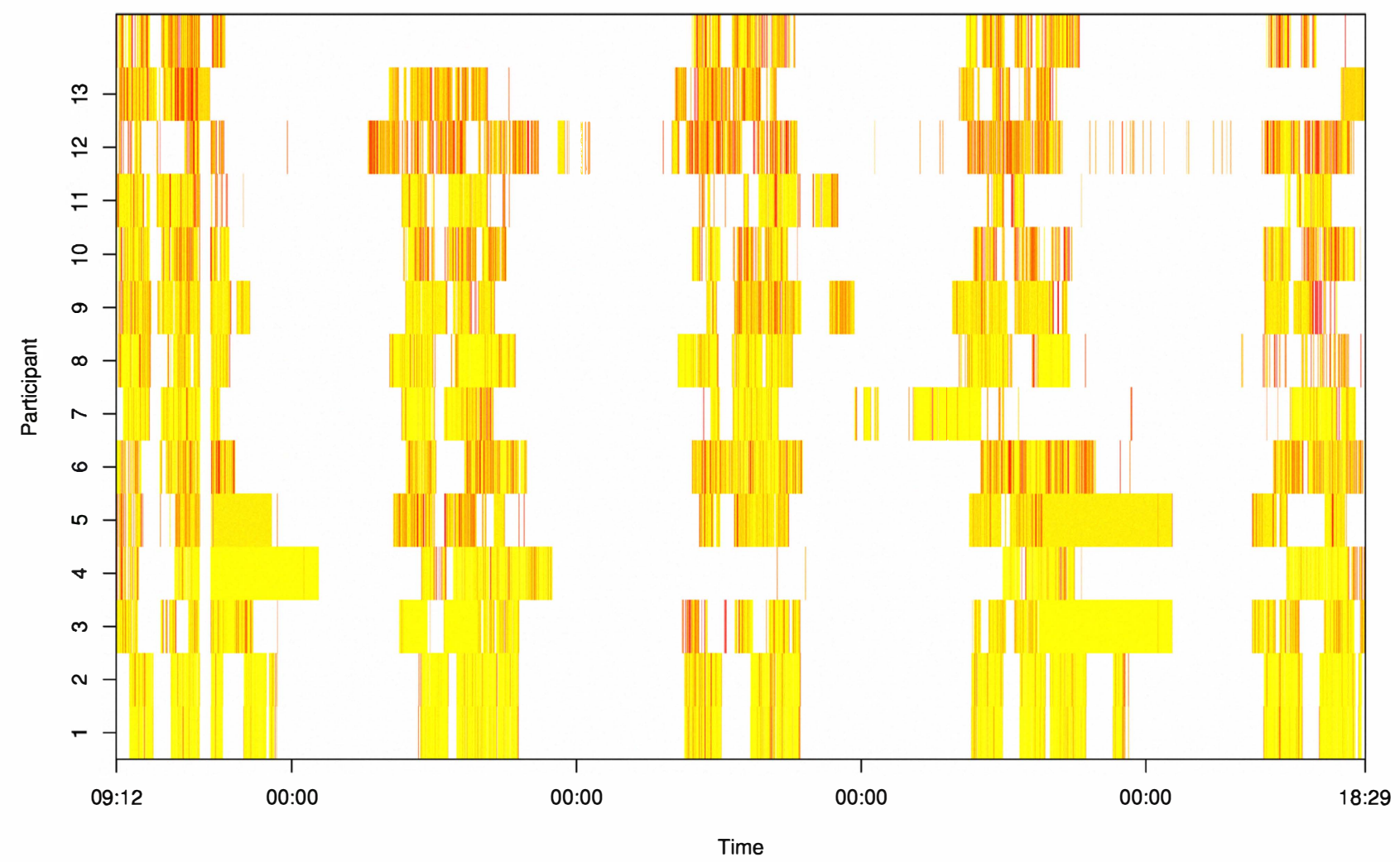

Figure 5. Visualisation of energy expenditure of occupants of the William Gates Building for one week. Darker colours indicate more intense activity.

[6] H. Besson, U. Ekelund, S. Brage, R. Luben, S. Bingham, K.-T. Khaw, and N. J. Wareham, "Relationship between subdomains of total physical activity and mortality," Medicine and science in sports and exercise, vol. 40, no. 11, pp. 190915, Nov 2008. [Online]. Available: http://meta.wkhealth.com/ $\mathrm{pt} / \mathrm{pt}$-core/template-journal/lwwgateway/media/landingpage. $\mathrm{htm}$ ?issn $=0195-9131 \&$ volume $=40 \&$ issue $=11 \&$ spage $=1909$

[7] S. T. Rassia, S. Hay, A. Beresford, and N. Baker, "Movement dynamics in office environments," SASBE 2009: Proceedings of the 3rd CIB International Conference on Smart and Sustainable Built Environments, 2009. [Online]. Available: http://www.cl.cam.ac.uk/ sjeh3/movement

[8] H. J. RALSTON, "Energy-speed relation and optimal speed during level walking," Internationale Zeitschrift für angewandte Physiologie, einschliesslich Arbeitsphysiologie, vol. 17, no. 4, pp. 277-83, Jan 1958. [Online]. Available: http: //www.ncbi.nlm.nih.gov/sites/entrez? $\mathrm{Db}=$ pubmed\&Cmd= Retrieve\&list_uids $=13610523 \&$ dopt=abstractplus

[9] A. Harter, A. Hopper, P. Steggles, A. Ward, and P. Webster, "The anatomy of a context-aware application," MobiCom '99: Proceedings of the 5th annual ACM/IEEE international conference on Mobile computing and networking, pp. 59-68, 1999. [Online]. Available: http://dx.doi.org/10.1145/313451. 313476

[10] M. Addlesee, R. Curwen, S. Hodges, J. F. Newman, P. Steggles, A. Ward, and A. Hopper, "Implementing a sentient computing system," IEEE Computer, vol. 34, no. 8, pp. 50-56, Sep 2001. [Online]. Available: http: //citeseer.ist.psu.edu/523300

[11] K. R. Westerterp, "Physical activity assessment with accelerometers," Int J Obes Relat Metab Disord, vol. 23 Suppl 3, pp. S45-9, Apr 1999. [Online]. Available: http: //www.ncbi.nlm.nih.gov/sites/entrez?Db=pubmed\&Cmd= Retrieve\&list_uids $=10368002 \&$ dopt $=$ abstractplus

[12] M. J. Mathie, A. C. F. Coster, N. H. Lovell, and B. G. Celler, "Accelerometry: providing an integrated, practical method for long-term, ambulatory monitoring of human movement," Physiological Measurement, vol. 25, p. R1, Apr 2004. [Online]. Available: http://www.iop.org/EJ/abstract/ 0967-3334/25/2/R01

[13] P. S. Freedson, E. Melanson, and J. Sirard, "Calibration of the computer science and applications, inc. 


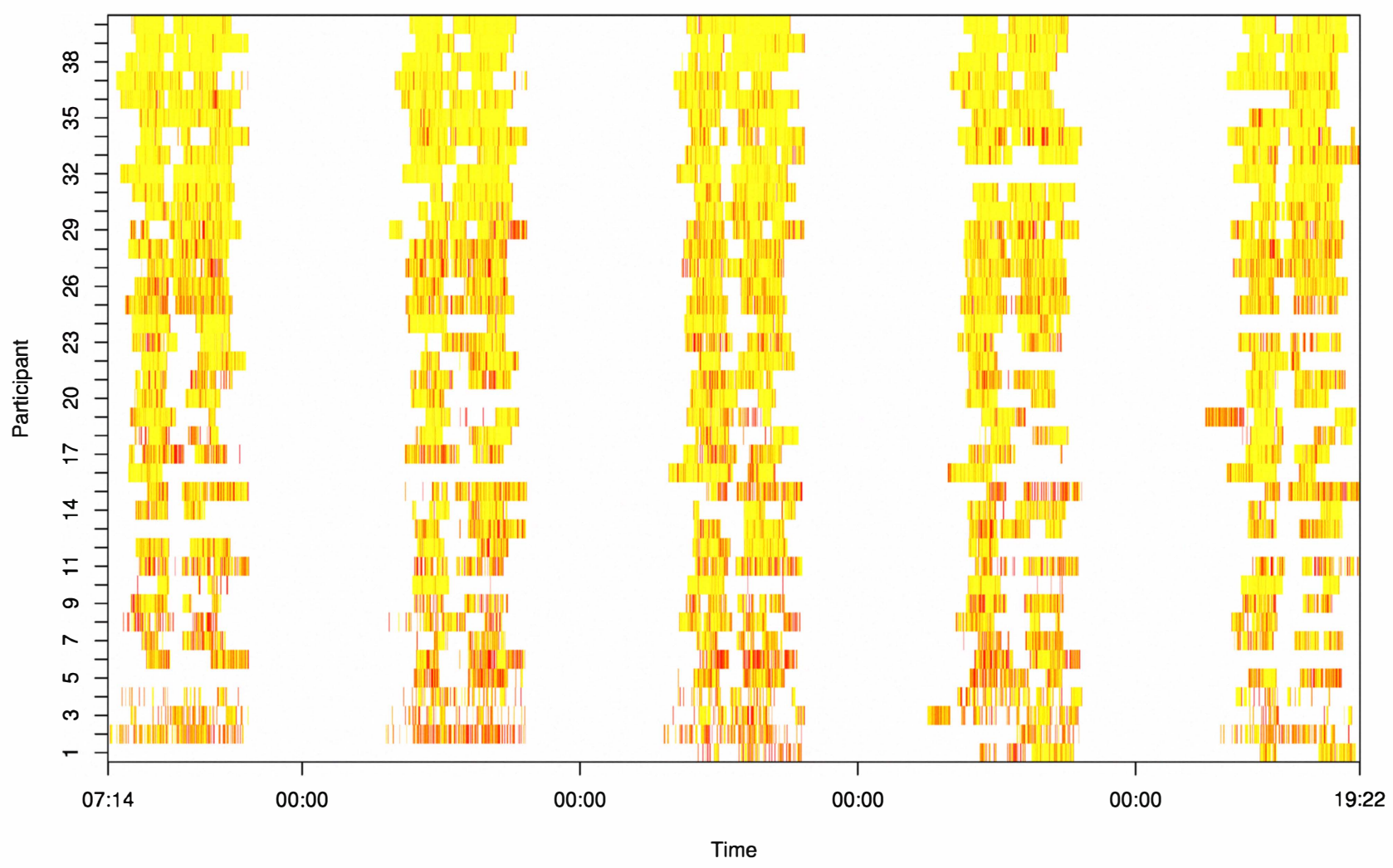

Figure 6. Visualisation of energy expenditure of top 40 occupants of Keynes House over a typical working week.

accelerometer," Medicine and science in sports and exercise, vol. 30, no. 5, pp. 777-81, May 1998. [Online]. Available: http://meta.wkhealth.com/pt/pt-core/ template-journal/lwwgateway/media/landingpage.htm?issn= 0195-9131\&volume $=30 \&$ issue $=5 \&$ spage $=777$

[14] A. Nilsson, S. Brage, C. Riddoch, S. A. Anderssen, L. B. Sardinha, N. Wedderkopp, L. B. Andersen, and U. Ekelund, "Comparison of equations for predicting energy expenditure from accelerometer counts in children," Scandinavian journal of medicine \& science in sports, vol. 18, no. 5, pp. 643-50, Oct 2008.

[15] S. Brage, N. Brage, P. W. Franks, U. Ekelund, and N. J. Wareham, "Reliability and validity of the combined heart rate and movement sensor actiheart," European journal of clinical nutrition, vol. 59, no. 4, pp. 561-70, Apr 2005. [Online]. Available: http://www.nature.com/ejcn/journal/v59/ n4/abs/1602118a.html

[16] K. Corder, S. Brage, and U. Ekelund, "Accelerometers and pedometers: methodology and clinical application," Current opinion in clinical nutrition and metabolic care, vol. 10, no. 5, pp. 597-603, Sep 2007.
[Online]. Available: http://meta.wkhealth.com/pt/pt-core/ template-journal/lwwgateway/media/landingpage.htm?issn= $1363-1950 \&$ volume $=10 \&$ issue $=5 \&$ spage $=597$

[17] J. Hightower and G. Borriello, "Location systems for ubiquitous computing," Computer, Jan 2001. [Online]. Available: http://ieeexplore.ieee.org/xpls/abs_all.jsp?arnumber=940014

[18] M. Mun, S. Reddy, K. Shilton, N. Yau, J. Burke, D. Estrin, M. Hansen, E. Howard, R. West, and P. Boda, "Peir, the personal environmental impact report, as a platform for participatory sensing systems research," Mobisys '09: Proceedings of the 7th international conference on Mobile systems, applications, and services, Jun 2009. [Online]. Available: http://portal.acm.org/citation.cfm?id=1555816.1555823

[19] S. Hay and R. Harle, "Bluetooth tracking without discoverability," LoCA 2009: Proceedings of the 4th International Symposium on Location and Context Awareness, 2009.

[20] P. Kurp, "Green computing," Communications of the $A C M$, vol. 51, no. 10, Oct 2008. [Online]. Available: http://portal.acm.org/citation.cfm?id=1400181.1400186 